\title{
Isolation and characterization of a female-specific DNA marker in the giant freshwater prawn Macrobrachium rosenbergii
}

\author{
T Ventura ${ }^{1,2}$, ED Aflalo ${ }^{1,2}$, S Weil ${ }^{1,2}$, K Kashkush $^{1}$ and A Sagi ${ }^{1,2}$ \\ ${ }^{1}$ Department of Life Sciences, Ben-Gurion University of the Negev, Beer-Sheva, Israel and ${ }^{2}$ National Institute for Biotechnology \\ in the Negev, Ben-Gurion University of the Negev, Beer-Sheva, Israel
}

\begin{abstract}
In this study, a female-specific DNA marker in the freshwater prawn Macrobrachium rosenbergii was identified through amplified fragment length polymorphism (AFLP). The AFLPderived sequence-characterized amplified region (SCAR) marker was tested in over 200 individuals, giving reproducible sex identification. Further molecular characterization of the sex-marker's genomic region ( $\sim 3 \mathrm{~kb}$ long) revealed the presence of tandem and inverted repeats. The $\sim 3-k b$ sequence was identified both in male and female prawns, but with subtle differences: a deletion of $3 \mathrm{bp}$ (present in female prawn but absent in male prawn) identified upstream of the SCAR marker sequence and two female-specific
\end{abstract}

single-nucleotide polymorphisms, both indicating that male prawns are homozygous, whereas female prawns are heterozygous in this locus. Fluorescent in situ hybridization showed the $\sim 3-k b$ sequence to be unique: to the best of our knowledge, this is the first report of a unique sex-specific sequence observed in situ in crustaceans. The sex-specific marker identified in $M$. rosenbergii may have considerable applied merit for crustacean culture in that it will enable the determination of genetic sex at early developmental stages when phenotypic differences are not identifiable.

Heredity (2011) 107, 456-461; doi:10.1038/hdy.2011.32; published online 27 April 2011

Keywords: crustacean; prawn; sex DNA marker; sex chromosomes; AFLP

\section{Introduction}

The development of molecular methods has led to the identification of molecular markers in a wide variety of species (Liu and Cordes, 2004; Granevitze et al., 2007). As a result, molecular markers and associated tools, such as genetic maps, are currently available for some crustacean species, particularly for shrimps. Molecular markers have been applied, for example, for analyzing the genetic diversity of wild shrimp resources (Xu et al., 2001), for strain and species identification in penaeids (Calo-Mata et al., 2009), and for parentage analysis in the black tiger shrimp Penaeus monodon (Jerry et al., 2006). In addition, linkage maps have been generated for several penaeid shrimp species, in most cases by using amplified fragment length polymorphism (AFLP) (Li et al., 2003; Zhang et al., 2007; Staelens et al., 2008). For prawns, however, no sex-specific markers have been identified, and to date $P$. monodon is the only decapod crustacean species for which sex-linked markers have enabled PCRbased differentiation of male from female prawns (Staelens et al., 2008).

Only very few invertebrate species are known to carry WZ sex chromosomes (Fuková et al., 2007), although

Correspondence: Professor A Sagi, Department of Life Sciences and the National Institute for Biotechnology, Ben-Gurion University of the Negev, POB 653, Beer-Sheva 84105, Israel.

E-mail: sagia@bgu.ac.il

Received 4 November 2010; revised 22 March 2011; accepted 24

March 2011; published online 27 April 2011 cumulative evidence suggests that WZ chromosomes are common in macruran decapod crustacean species (crayfish, lobsters, spiny lobsters, shrimps and prawns) (Staelens et al., 2008). Evidence for type and/or presence of sex chromosomes is drawn from physical mapping of sex-specific sequences, from sex-linked linkage maps, from karyological studies and from crosses of sexreversed individuals (Legrand et al., 1987; Sagi and Cohen, 1990; Griffiths and Orr, 1999; Li et al., 2003; Parnes et al., 2003). In Crustacea of the order Decapoda, karyotype studies have revealed distinguishable sex chromosomes only in crabs (Brachyura) and hermit crabs (Anomura), as reviewed by Parnes et al. (2003). In all nine species reviewed, the male prawn was found to be the heterogametic sex, bearing XY chromosomes. In the few macruran species for which karyotype studies are available, including those in the giant freshwater prawn Macrobrachium rosenbergii (Justo et al., 1991), sex chromosomes are morphologically indistinguishable from autosomes. It is believed that in $M$. rosenbergii, as in other macruran species, the female prawn is the heterogametic sex, bearing WZ sex chromosomes, whereas the male prawn is the homogametic sex, bearing $\mathrm{ZZ}$ sex chromosomes. Data supporting this premise may be taken from two sex reversal studies: In one study, mating of sex-reversed male prawns with normal male prawns produced all-male $(Z Z \times Z Z)$ progeny (Sagi and Cohen, 1990; Aflalo et al., 2006). In the other study, the progeny of sex-reversed female prawns mated with normal female prawns $(\mathrm{WZ} \times \mathrm{WZ})$ exhibited a sex ratio skewed to female prawns (Malecha et al., 1992). 
In this study, we aimed at identifying sex-specific markers in $M$. rosenbergii by using AFLP. We isolated the first sex-specific genomic marker for this species and successfully converted it into a female-specific sequencecharacterized amplified region (SCAR) marker, thereby enabling PCR-based sex identification based on several female-specific polymorphic features. Fluorescent in situ hybridization (FISH) provided proof that the genomic region in the vicinity of the sex-marker constitutes a unique sequence, as it appears only in one locus in the genome. On the basis of our findings, we posit that the sex marker is probably located in the sex chromosome, in a locus common to the $\mathrm{Z}$ and $\mathrm{W}$ chromosomes.

\section{Materials and methods}

\begin{abstract}
Animals
The broodstock for the $M$. rosenbergii prawns currently being cultured in Israeli aquafarms was imported into Israel from Hawaii approximately two decades ago. The prawns used for this study were maintained at Ben-Gurion University of the Negev under the following conditions: Food comprising shrimp pellets (Rangen Inc., Buhl, ID, USA; 30\% protein) was supplied ad libitum three times a week. Water quality was assured by circulating the entire volume through a bio-filter maintaining all the water physicochemical parameters as described before (Khalaila et al., 2002). Each egg-berried female prawn was transferred to a separate closed 100-L tank containing 12-16 ppt salt water, which was circulated through a $100-\mu \mathrm{m}$ mesh net. After the brood had hatched, the female prawn was removed from the tank, and the newly hatched larvae were maintained according to the protocol devised by Uno and Chin Soo (1969) and fed with Artemia nauplii daily. Prawns were also sampled from two other populations, one grown by Ms Ayana Perlberg of the Dor Research Center and the other by Dr Shmuel Parnes of the ornamental aquaculture company, Colors (Moshav Hatzeva, Israel).
\end{abstract}

\section{Genomic DNA extraction}

Genomic DNA was extracted from frozen tail muscle tissue with a Gentra Puregene Tissue Kit (Qiagen, Chatsworth, CA, USA) or REDExtract-N-Amp Tissue PCR Kit (Sigma, St Louis, MI, USA), according to the manufacturer's instructions.

\section{AFLP reaction}

AFLP was carried out according to the method of Kashkush et al. (2001). Briefly, $0.5 \mu \mathrm{g}$ of genomic DNA from three male and three female individuals was digested with EcoRI and MseI and ligated to adaptors. The digestion/ligation reaction was carried out at $37^{\circ} \mathrm{C}$ for $2 \mathrm{~h}$. The pre-selective PCR was carried out with primers complementary to the core adaptor primer, and selective amplification was carried out with $\left[\gamma_{-}{ }^{32} \mathrm{P}\right] \mathrm{ATP}$ end-labeled EcoRI primer and other primers complementary to the MseI adaptor sequence, with three additional random nucleotides to each primer. The PCR products were mixed with $20 \mu \mathrm{l}$ of formamide dye $(98 \%$ formamide, $10 \mathrm{mM}$ EDTA, $0.1 \%\left(\mathrm{w} \mathrm{v}^{-1}\right)$ bromophenol blue, $0.1 \%\left(\mathrm{w} \mathrm{V}^{-1}\right)$ xylene cyanol), heated at $95^{\circ} \mathrm{C}$ for $5 \mathrm{~min}$ and separated on $5 \%$ denaturing polyacrylamide standard sequencing gel (40\% acrylamide:bisacrylamide at a 1:20 ratio, $7.5 \mathrm{M}$ urea, $1 \times$ Tris borate EDTA-TBE buffer, $43 \mathrm{~cm}$ long) at $55 \mathrm{~W}$ for $1.5 \mathrm{~h}$ (after $40 \mathrm{~min}$ of prerun under the same conditions). Gels were then dried on a gel drier for $2 \mathrm{~h}$ at $70{ }^{\circ} \mathrm{C}$ and exposed to X-ray film (Fuji, Tokyo, Japan) for about $16 \mathrm{~h}$ at room temperature. Unique male and female bands were extracted from the gel, re-amplified under the same PCR conditions as used for selective amplification, cloned and sequenced.

\section{Sequence-characterized amplified region}

Specific primers were designed to amplify each of the isolated AFLP bands. PCR was performed with $100 \mathrm{ng}$ of genomic DNA, $1 \mu \mathrm{M}$ of forward primer and $1 \mu \mathrm{M}$ of reverse primer (primer sequences are available upon request), $12.5 \mu$ l of Ready Mix REDTaq (Sigma, St Louis, MO, USA) and water to a final volume of $25 \mu \mathrm{l}$. PCR conditions were: $30 \mathrm{~s}$ at $94{ }^{\circ} \mathrm{C}, 30 \mathrm{~s}$ at $56^{\circ} \mathrm{C}$ and $60 \mathrm{~s}$ at $72{ }^{\circ} \mathrm{C}$, followed by 35 cycles. PCR products were electrophoresed on a $2 \%$ agarose gel and visualized under ultraviolet light with ethidium bromide.

\section{Genome walking}

Genome walking was carried out using DNA Walking SpeedUp Premix Kit (Seegene, Rockville, MD, USA), according to the manufacturer's specifications.

\section{Fluorescent in situ hybridization}

FISH was carried out as described by Dzikowski et al. (2007), with the exceptions that the prawn sample was prepared according to Justo et al. (1991) and that the probe used was the PCR probe of nucleotides 1-2003 of the $\sim 3 \mathrm{kbp}$ fragment identified (primers available upon request). Samples were prepared from several male and female gonads, and the signal was scored for a total of 74 nuclei that could be distinguished morphologically. On the basis of the nucleus morphology, the signals were scored positively for one dot (haploid cells) or two dots (diploid cells). In some cases, two double-dotted spots were also scored.

\section{Sequence analysis}

Sequence annotation relied on the BLAST 2.0 package from NCBI (http://www.ncbi.nlm.nih.gov/BLAST/) and from the Institute for Genomic Research (http:/ / tigrblast.tigr.org/tgi/). Repetitive sequences were identified by the self-blast tool (http://www.proweb.org/ proweb/Tools/selfblast.html) and the tandem repeats finder (http://tandem.bu.edu/trf/trf.html) (Benson, 1999). Open reading frames along the sequence were viewed with the NEBcutter (http://tools.neb.com/NEB cutter2/) (Vincze et al., 2003). Indel identification in uncloned sequences was obtained by Shift Detector (http: / / cowry.agri.huji.ac.il/cgi-bin/frshplus.cgi) (Seroussi et al., 2002).

\section{Results and discussion}

\section{Sex-specific AFLP markers}

The AFLP assay is an ideal system for the identification of sex-specific DNA markers, because it requires only a small amount of genomic DNA for the study of hundreds of genomic loci in a relatively short time and, more importantly, in an unbiased manner (Vos et al., 1995). In addition, AFLP is ideal for studying poorly deciphered 
a

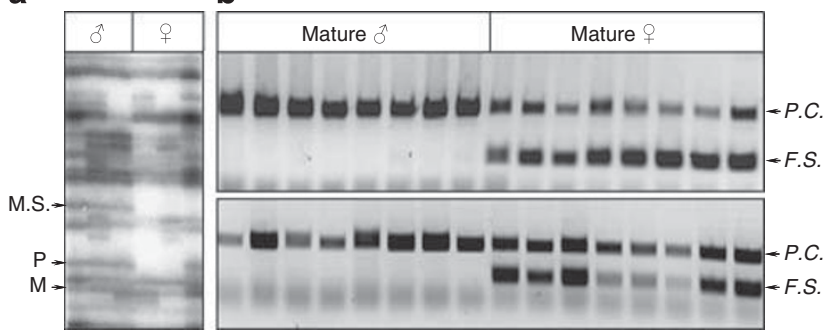

Figure 1 Amplified fragment length polymorphism (AFLP) screening for sex-specific genomic sequences in $M$. rosenbergii and PCR of genomic DNA extracted from mature $M$. rosenbergii. (a) AFLP profiles of three male and three female mature $M$. rosenbergii prawns obtained with a combination of Eco-NN- and Mse-NN-selective primers (' $N$ ' represents a randomly picked nucleotide). Representative monomorphic (M), polymorphic (P) and male-specific (MS) sequences are indicated by arrows. (b) The female-specific genomic sequence (FS) is amplified in all mature female specimens and is absent in all mature male specimens, as shown by PCR. Positive control (PC), which was derived from an AFLP monomorphic band, is present in all specimens. Primer sequences are available upon request.

genomes, such as the genome of the freshwater prawn $M$. rosenbergii, because it does not require any previous knowledge about the genomes. Using 18 primer combinations in the AFLP reaction, we screened six prawnsthree males and three females-with the aim to identify sex-specific markers. A representative AFLP product is shown in Figure 1a. Of a total of 1795 clearly distinguishable AFLP bands screened, 65 were specific to male prawns, and 87 specific to female prawns. The sexspecific bands could be classified into four groups: (1) 12 bands were present in all three male prawns and absent in all three female prawns; (2) 16 bands were present in all three female prawns, but absent in all three prawns; (3) 54 bands were present in one or two male prawns and absent in all three prawns; and (4) 71 bands were present in one or two female prawns, but absent in all three prawns. These data suggested that the bands classified into groups 1 and 2 could be sex specific and hence could serve as sex-specific markers.

Validation of the sex-specific bands by site-specific PCR To convert AFLP markers into SCAR markers, the 16 female-specific and the 12 male-specific bands identified above were extracted from the gel and sequenced. Specific primers were designed for each sequence and used in the PCR amplification with DNA from four male prawns and four female prawns as the template. In 27 of the cases, monomorphic PCR bands were observed in both male and female cases, thereby making it difficult to convert these bands into SCAR markers. In one case, however, a PCR product ( $\sim 140 \mathrm{bp}$ long) was amplified for the female prawns, but was absent for the male prawns (Figure 1b; FS). This SCAR marker was tested in over 200 individuals drawn at random from the three populations of prawns described above (see Materials and methods), with $100 \%$ success in the identification of female prawns (see examples in Figure $1 \mathrm{~b}$ and Supplementary Figure S1). Our success rate (1 out of 28) for converting an AFLP marker into a SCAR marker falls in the range of the success rates reported in previous studies in salmonids-one out of 15 reported by Felip et al. (2005) and one out of 52 reported by Brunelli and
Thorgaard (2004). We posit that it might be possible to convert some of the other 27 sex-specific AFLP bands into SCAR markers by using genome walking to reveal the sequences spanning the regions outside the AFLP marker, as was carried out for the male-sterility marker in the plant Brassica napus (Zeng et al., 2009) and in potato; in the latter study, genome walking facilitated the successful conversion of six of 10 AFLP markers to SCAR markers (Brugmans et al., 2003). Genome walking was necessary in the above six instances owing to the fact that the single-nucleotide polymorphisms (SNPs) resided in the restriction site. In our study, the finding of a female-specific sex marker-rather than a male-specific marker-was not surprising, as it is believed that in $M$. rosenbergii sex determination is governed by a WZ/ZZ mechanism (Sagi and Cohen, 1990).

\section{Molecular characterization of the sex-specific marker and its genomic vicinity}

DNA sequencing of genomic regions flanking the SCAR marker $(\sim 3 \mathrm{~kb})$ in female prawns revealed the presence of seven tandem repeats, each of $88 \mathrm{bp}$, residing $83-698 \mathrm{bp}$ upstream of the sex-specific marker. Each tandem repeat started with a 12-bp palindrome sequence (Figure 2a). In addition, two 11-bp inverted repeats were identified further upstream (starting at 432 and $794 \mathrm{bp}$ after the tandem repeats), and yet another one $614 \mathrm{bp}$ downstream to the sex-specific marker (Figure 2a). Another set of primers (Figure 2b, P1 and P2) flanking the SCAR marker was used for amplifying a larger DNA fragment of $\sim 340 \mathrm{bp}$ (Figure 2b). Surprisingly, PCR amplification of the 340-bp fragment produced positive products for both male and female prawns. Sequence analysis of this fragment revealed that the original AFLP sex-marker included two female-specific SNPs in the primer location (P3 in Figure 2b), which enabled the production of the SCAR female-specific marker (Figure 1b). Moreover, three nucleotides located between the SCAR sequence and the tandem repeats sequence, within the 340-bp amplified fragment, were present in all the cloned male sequences, but absent in some of the female clones (Figure $2 b, \ldots$ 岁 ). PCR amplification using a forward primer designed on the basis of femalespecific deletion (P4 in Figure $2 \mathrm{~b}$ ) and a reverse primer downstream of the SCAR sequence ( $\mathrm{P} 2$ in Figure $2 b$ ) produced positive products only in female prawns (Figure 3a, top panel). However, PCR amplification using a forward primer that included the three nucleotides (P5 in Figure 2b) and a reverse primer downstream of the SCAR sequence ( $\mathrm{P} 2$ in Figure $2 \mathrm{~b}$ ) produced positive products for both male and female prawns (Figure 3a, bottom panel). Un-cloned sequences amplified from DNA of five female and five male prawns by using primers P2 and P5 were obtained. For all five male prawns, the sequences resulted in single peaks (Figure $3 b$, left bottom), whereas for all five female individuals, the sequences contained overlapping peaks (Figure 3b, left top). The female sequences were treated with Shift Detector software (Seroussi et al., 2002), where the 3-bp deletion in female prawns was detected with a probability score of $1.53 \mathrm{e}^{-05}$. In addition, sequencing of un-cloned female-specific PCR products obtained in the previous PCR (using primers P4 and P2; Figure 2b) produced single peaks, whereas no amplification was found for male prawns (Figure $3 \mathrm{~b}$ right). 


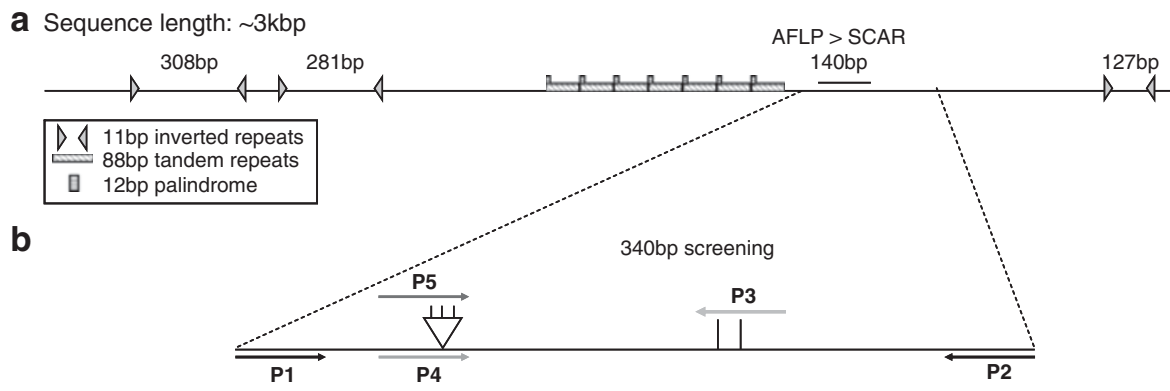

Figure 2 Schematic overview of the original sex-specific sequence found by amplified fragment length polymorphism (AFLP) and the further upstream and downstream sequence features obtained by genome walking and sequence characterization. (a) The $\sim 3-\mathrm{kbp}$ sequence obtained by AFLP and genome walking. Seven tandem repeats of $88 \mathrm{bp}$, each starting with a 12-bp palindrome, are indicated by striped rectangles, as are three 11-bp inverted repeats (indicated by triangles). (b) A 340-bp sequence within the 3-kbp sequence obtained by genomic walking in the area surrounding the original AFLP product was amplified both in male and female prawns. In this 340-bp sequence, two female-specific SNPs were identified ( $\downarrow$ ), which enabled the female-specific amplification of the SCAR marker. An insertion of three nucleotides

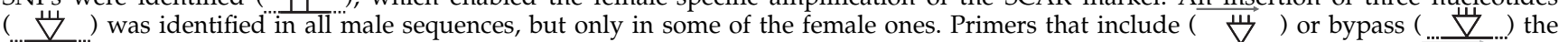
insertion were designed. A full color version of this figure is available at the Heredity journal online.

a
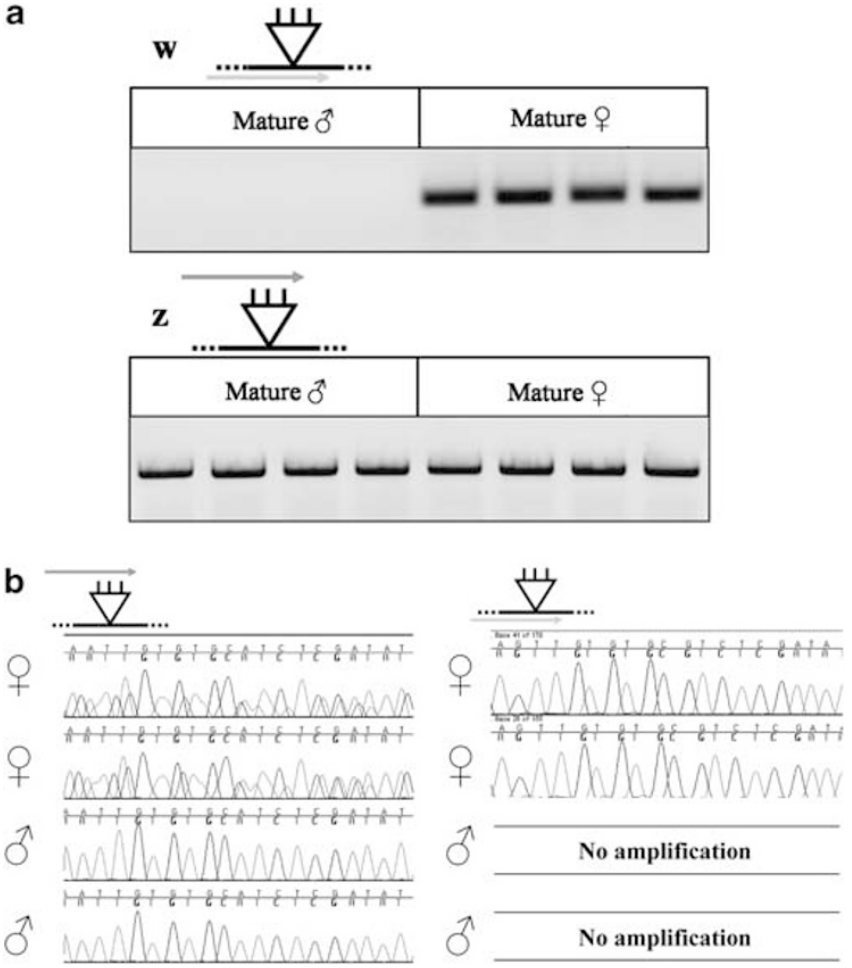

Figure 3 Gender dimorphic genomic patterns in the region of the original amplified fragment length polymorphism (AFLP) product. The 3-bp insertion found in the proximity of the AFLP marker was further analyzed by using primers that bypass or include the insertion against a common reverse primer (P2 in Figure 2a). (a) The primer that bypasses the insertion $(\ldots, \vec{\nabla})$ amplified a fragment only in female prawns (top), but the primer that includes the insertion ( $\ddot{\nabla}$ ) amplified a fragment both in male and female prawns (bottom), leading to the hypothesis that this genomic sequence is homozÿgous in male prawns (ZZ) and heterozygous in female prawns (WZ). The primer that bypasses the insertion amplified only the $\mathrm{W}$ fragment, which is unique to female prawns, and the primer that includes the insertion amplified the $\mathrm{Z}$ fragment common to both male and female prawns. (b) When the primer that includes the insertion was used, un-cloned sequences amplified from both male and female prawns showed that the male sequence produced single peaks and the female sequence included overlapping peaks; this finding thus confirmed that female prawns are heterozygous in this region. When the primer that bypasses the insertion was used, the female sequences produced single peaks, and there was no amplification in the male prawns. Primer sequences are available upon request. A full color version of this figure is available at the Heredity journal online.

In summary, we used several methodologies, all of which provided support for the conclusion that female prawns are heterozygous and male prawns are homozygous in the locus of the sex marker with subtle differences, such as a 3-bp indel and two SNPs between the two female alleles. The most solid proof for the above notion derives from the results of our cloning experiments: we cloned and sequenced both the female-specific allele and the female/male shared allele. To confirm the indel identified in the female cloned sequences, un-cloned female sequences were investigated with Shift Detector software (Seroussi et al., 2002), which 

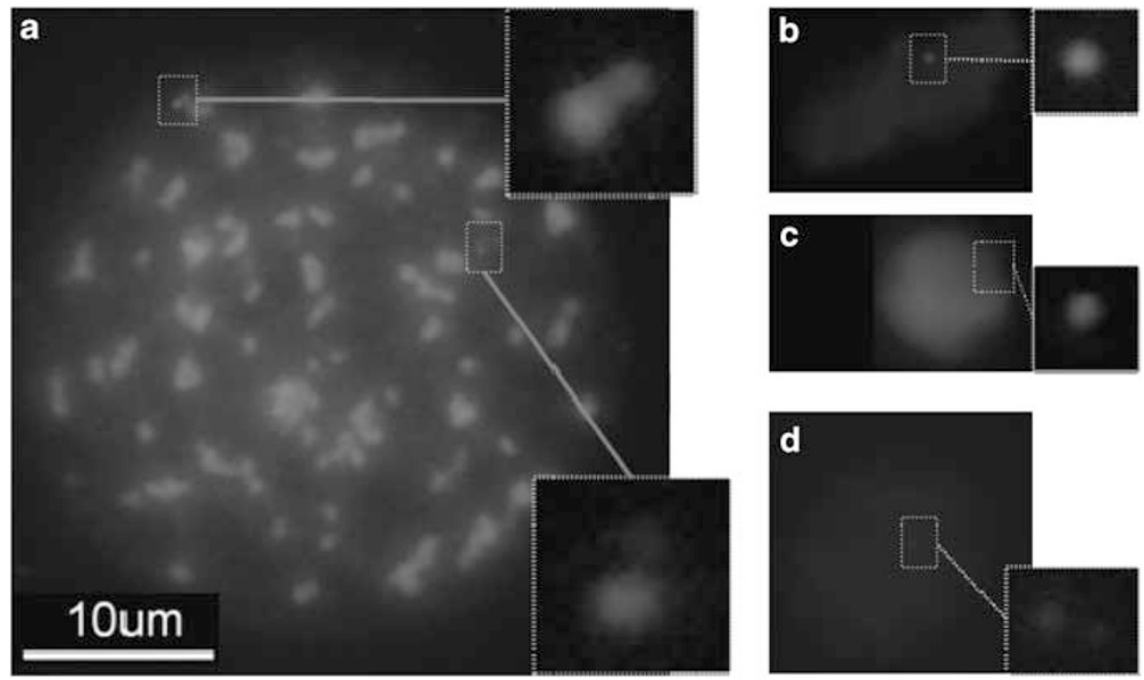

Figure 4 Fluorescent in situ hybridization of the sex-specific marker. Representative hybridizations from several cell types are shown. Nuclei are stained with 4',6-diamidino-2-phenylindole (blue), and red indicates the specific signal: (a) Two double-dotted spots in a nucleus in first meiotic division (2n4c). (b) A single dot (1n1c) of a spermatozoa and a spermatid (c). (d) Two-dotted spot (1n2c) in a secondary spermatocyte (bar $=10 \mu \mathrm{m}$ ). A full color version of this figure is available at the Heredity journal online.

successfully identified the indel as present in the cloned sequences.

The identification of new alleles based on sequencespecific design of primers for PCR has been reported previously (Wang et al., 1995; Chung et al., 2003). It is worth noting that in the tiger shrimp P. monodon, the sex-linked SCAR marker, which resides on the sex chromosome, is also based on a short indel in the female-specific allele (Staelens et al., 2008). To assess the copy number of the region, FISH was carried out on cells extracted from several male gonads; $2 \mathrm{~kb}$ out of the $\sim 3 \mathrm{~kb}$ first identified in female prawns was used as a probe. The high similarity between the female-specific allele and the female/male shared allele enabled us to perform FISH for the male prawns, for which there is a known protocol for cell extraction (Justo et al., 1991). In all cases in which the field was clean of background signals, the nuclei were scored according to their morphology and the number of signal spots. In four cases, there were two double-dotted spots in cells in their first meiotic division (2n4c; Figure 4a). Of the 47 instances of single dots (1n1c), 24 were spermatozoa (Figure $4 \mathrm{~b}$ ) and 23 were spermatids (Figure 4c). Twentythree double-dotted spots (1n2c) were found in secondary spermatocytes (Figure 4d). The above results taken together indicate that the genomic region of the sexspecific marker constitutes a unique sequence in $M$. rosenbergii. The short unique marker sequence of $\sim 3 \mathrm{~kb}$ obtained in this study should be carefully investigated in other populations with the ultimate aim of devising a global sex-specific marker for $M$. rosenbergii that will enable the sex identification of individuals from unrelated populations, as was carried out for a DNA sex marker identified in the three-spined stickleback (Griffiths et al., 2000). In the basket willow (Salix viminalis), on the other hand, researchers identified sex-linked DNA markers derived from unrelated families (and therefore genetically variable), and thus concluded that these sex-linked markers represent a wide natural population rather than a limited experimental group (Semerikov et al., 2003).

\section{Significance of identifying sex-specific markers in} M. rosenbergii

The significance of sex identification in $M$. rosenbergii lies in the importance of this species in aquaculture. As male prawns of the species grow faster than female prawns, it is not uncommon to find farms that hand segregate the male prawns for marketing and discard the female prawns, a method proven to increase yield and income for the growers by $\sim 60 \%$ (Nair et al., 2006). The bimodal growth pattern of $M$. rosenbergii, that is, male prawns growing faster than female prawns (Sagi et al., 1986), has led to the elaboration of a biotechnology for monosex population production (Aflalo et al., 2006). This biotechnology relies on a fully functional sex reversal of normal male prawns into neo-female prawns to produce all-male progeny. In the implementation of such a biotechnology, the availability of sex-specific markers could shorten the time needed for progeny testing, which is a crucial, timeconsuming and labor-intensive stage in the proposed biotechnology. The nature of the genomic region revealed in this study (a unique sequence, dimorphic in female prawns and monomorphic in male prawns) has led us to hypothesize that this region resides in the sex chromosome in a locus common to the $\mathrm{W}$ and $\mathrm{Z}$ chromosomes. This idea is in keeping with the heterogametic female theory of Katakura (1989). However, as the sex chromosome of $M$. rosenbergii is not distinguishable morphologically (Justo et al., 1991), further research is needed to verify this notion.

\section{Conflict of interest}

The authors declare no conflict of interest.

\section{Acknowledgements}

We would like to thank Mr Shahar Shimshon for prawn maintenance in our facilities at BGU and Ms Ayana Perlberg for housing the prawns at Dor Research Center. We also thank Dr Shmuel Parnes of the ornamental 
aquaculture company 'Colors' for allowing us to sample prawns grown in his facilities, and Dr Ron Dzikowski of the Hebrew University of Jerusalem for his guidance with the FISH technique. This study was supported in part by grants from the National Institute for Biotechnology in the Negev (NIBN) and US-AIDCDR (no. 13025).

\section{References}

Aflalo ED, Hoang TTT, Nguyen VH, Lam Q, Nguyen DM, Trinh QS et al. (2006). A novel two-step procedure for mass production of all-male populations of the giant freshwater prawn Macrobrachium rosenbergii. Aquaculture 256: 468-478.

Benson G (1999). Tandem repeats finder: a program to analyze DNA sequences. Nucleic Acids Res 27: 573-580.

Brugmans B, van der Hulst RGM, Visser RGF, Lindhout $P$, van Eck HJ (2003). A new and versatile method for the successful conversion of AFLP markers into simple single locus markers. Nucleic Acids Res 31: e55.

Brunelli JP, Thorgaard GH (2004). A new Y-chromosomespecific marker for pacific salmon. Trans Am Fish Soc 133: 1247-1253.

Calo-Mata P, Pascoal A, Fernandez-No I, Bohme K, Gallardo JM, Barros-Velazquez J (2009). Evaluation of a novel 16S rRNA/tRNAVal mitochondrial marker for the identification and phylogenetic analysis of shrimp species belonging to the superfamily Penaeoidea. Anal Biochem 391: 127-134.

Chung C, Leib SR, Fraser DG, Ellis SA, McGuire TC (2003). Novel classical MHC class I alleles identified in horses by sequencing clones of reverse transcription-PCR products. Eur I Immunogenet 30: 387-396.

Dzikowski R, Li F, Amulic B, Eisberg A, Frank M, Patel S et al. (2007). Mechanisms underlying mutually exclusive expression of virulence genes by malaria parasites. EMBO Rep 8: 959-965.

Felip A, Young WP, Wheeler PA, Thorgaard GH (2005). An AFLP-based approach for the identification of sex-linked markers in rainbow trout (Oncorhynchus mykiss). Aquaculture 247: 35-43.

Fuková I, Traut W, Vítková M, Nguyen P, Kubíèková S, Marec F (2007). Probing the W chromosome of the codling moth, Cydia pomonella, with sequences from microdissected sex chromatin. Chromosoma 116: 135-145.

Granevitze Z, Blum S, Cheng H, Vignal A, Morisson M, Ben-Ari $\mathrm{G}$ et al. (2007). Female-specific DNA sequences in the chicken genome. J Hered 98: 238-242.

Griffiths R, Orr K (1999). The use of amplified fragment length polymorphism (AFLP) in the isolation of sex-specific markers. Mol Ecol 8: 671-674.

Griffiths R, Orr KL, Adam A, Barber I (2000). DNA sex identification in the three-spined stickleback. J Fish Biol 57: 1331-1334.

Jerry DR, Evans BS, Kenway M, Wilson K (2006). Development of a microsatellite DNA parentage marker suite for black tiger shrimp Penaeus monodon. Aquaculture 255: 542-547.

Justo CC, Murofushi M, Aida K, Hanyu I (1991). Karyological studies on the freshwater prawn Macrobrachium rosenbergii. Aquaculture 97: 327-334.

Kashkush K, Jinggui F, Tomer E, Hillel J, Lavi U (2001). Cultivar identification and genetic map of mango (Mangifera indica). Euphytica 122: 129-136.

Katakura Y (1989). Endocrine and genetic control of sex differentiation in the malacostracan Crustacea. Invertebr Reprod Dev 16: 177-182.
Khalaila I, Manor R, Weil S, Granot Y, Keller R, Sagi A (2002). The eyestalk-androgenic gland-testis endocrine axis in the crayfish Cherax quadricarinatus. Gen Comp Endocrinol 127: 147-156.

Legrand JJ, Legrand-Hamelin E, Juchault P (1987). Sex determination in Crustacea. Biol Rev Camb Philos Soc 62: 439-470.

Li YT, Byrne K, Miggiano E, Whan V, Moore S, Keys S et al. (2003). Genetic mapping of the kuruma prawn Penaeus japonicus using AFLP markers. Aquaculture 219: 143-156.

Liu ZJ, Cordes JF (2004). DNA marker technologies and their applications in aquaculture genetics. Aquaculture 238: 1-37.

Malecha SR, Nevin PA, Ha P, Barck LE, Lamadrid-Rose Y, Masuno S et al. (1992). Sex-ratios and sex-determination in progeny from crosses of surgically sex-reversed freshwater prawns, Macrobrachium rosenbergii. Aquaculture 105: 201-218.

Nair CM, Salin KR, Raju MS, Sebastian M (2006). Economic analysis of monosex culture of giant freshwater prawn (Macrobrachium rosenbergii De Man): a case study. Aquacult Res 37: 949-954.

Parnes S, Khalaila I, Hulata G, Sagi A (2003). Are intersex crayfish Cherax quadricarinatus (Von Martens) genetically females? Genet Res 82: 107-116.

Sagi A, Cohen D (1990). Growth, maturation and progeny of sex-reversed Macrobrachium rosenbergii males. World Aquacult 21: 87-90.

Sagi A, Ráanan Z, Cohen D, Wax Y (1986). Production of Macrobrachium rosenbergii in monosex population: yield characteristics under intensive monoculture conditions in cages. Aquaculture 51: 265-275.

Semerikov V, Lagercrantz U, Tsarouhas V, Ronnberg-Wastljung A, Alstrom-Rapaport C, Lascoux M (2003). Genetic mapping of sex-linked markers in Salix viminalis L. Heredity 91 293-299.

Seroussi E, Ron M, Kedra D (2002). ShiftDetector: detection of shift mutations. Bioinformatics 18: 1137-1138.

Staelens J, Rombaut D, Vercauteren I, Argue B, Benzie J, Vuylsteke M (2008). High-density linkage maps and sexlinked markers for the black tiger shrimp (Penaeus monodon). Genetics 179: 917-925.

Uno Y, Chin Soo K (1969). Larval development of Macrobrachium rosenbergii (de Man) reared in the laboratory. J Tokyo Univ Fish 55: 179-190.

Vincze T, Posfai J, Roberts RJ (2003). NEBcutter: a program to cleave DNA with restriction enzymes. Nucleic Acids Res 31: 3688-3691.

Vos P, Hogers R, Bleeker M, Reijans M, van de Lee T, Hornes M et al. (1995). AFLP: a new technique for DNA fingerprinting. Nucleic Acids Res 23: 4407-4414.

Wang $\mathrm{H}$, Tokunaga $\mathrm{K}$, Ishikawa $\mathrm{Y}$, Lin L, Kashiwase $\mathrm{K}$, Nakajima $F$ et al. (1995). A new HLA-C allele, CWl403, associated with HLA-B44 in Japanese. Hum Immunol 43: 295-300.

Xu Z, Primavera JH, de la Pena LD, Pettit P, Belak J, AlcivarWarren A (2001). Genetic diversity of wild and cultured black tiger shrimp (Penaeus monodon) in the Philippines using microsatellites. Aquaculture 199: 13-40.

Zeng F, Yi B, Tu J, Fu T (2009). Identification of AFLP and SCAR markers linked to the male fertility restorer gene of pol CMS (Brassica napus L.). Euphytica 165: 363-369.

Zhang L, Yang C, Zhang Y, Li L, Zhang X, Zhang Q et al. (2007). A genetic linkage map of Pacific white shrimp (Litopenaeus vannamei): sex-linked microsatellite markers and high recombination rates. Genetica 131: 37-49. 